\title{
"Por más que yo trabaje, trato de acomodar todo a mi hijo": Usos de la visualización de líneas de tiempo para comprender desigualdades de género en la academia
}

"Regardless of how much I work, I try to make adjustments for my son's sake": Uses of timeline visualizations to understand gender inequalities in academia

Francisca Ortiz Ruiz

The University of Manchester, Reino Unido

Camila Moyano Dávila

Daniela Moyano Dávila

Pontificia Universidad Católica de Chile, Chile

RESUMEN Este artículo tiene por objetivo dar cuenta cómo, en términos metodológicos, las trayectorias de vida en su componente cualitativo entregan información valiosa aprovechada mediante el uso de la visualización de líneas de tiempo. En general estas han sido ampliamente utilizadas por estudios cuantitativos, y esta investigación se develará su utilidad en un proyecto cualitativo en la recolección de datos, análisis y posterior presentación. Esto, debido a que una de las características principales de la visualización de las líneas de tiempo es emplazar la información a escala humana, y así promover la plausibilidad de las comparaciones entre casos. Así este artículo espera mostrar la utilidad de la visualización de datos cualitativos a través de su ejemplificación en la comparación de cuatro líneas biográficas de académicos de una universidad chilena. Se utiliza una definición particular de trayectoria académica, a saber, la imbricación de aspectos laborales, familiares y de pareja que se desarrollan en el contexto de la academia. Con esta definición como punto de partida, se articularon estas líneas de tiempo con las biografías narrativas de los/as académicos/as. La utilización de colores, símbolos según el evento, y citas de las entrevistas en cada uno de los momentos en las líneas biográficas, permite dar cuenta que, en el caso de los hombres, la trayectoria laboral sigue una linealidad progresiva y 
distanciada de la trayectoria familiar. Al contrario, en el caso de las mujeres, su trayectoria laboral se ve puesta en evaluación y/o tensionada respecto de la trayectoria familiar. Así se concluye, que la visualización de las líneas de tiempo es una herramienta flexible pues puede actuar como una ayuda memoria a los/as entrevistados/as durante el trabajo de terreno. Además, permite dar cuenta de aspectos subjetivos para ser trabajados analíticamente, hacer comparaciones, y mostrar de una forma amable la información para diversas audiencias y grupos de investigación.

PALABRAS CLAVE Visualización de datos; enfoque biográfico; metodología cualitativa; trayectorias académicas; comparación de género; subjetividad.

ABSTRACT The objective of this article is to show how, in methodological terms, life trajectories in their qualitative component seem to provide information where data visualization - usually used by quantitative studies - could contribute enough to the data collection, analysis and the subsequent presentation. One of the main features of data visualization is that it would allow placing the information on a human scale, which would make comparing cases a more plausible process to perform. In this sense, this article hopes to show the usefulness of the visualization of qualitative data based on the comparison of four biographical lines of chemistry academics of a Chilean university. We use a particular definition of academic trajectory, namely, the overlap of work, family, personal and couple aspects that are developed in the academic context. With this definition as a starting point, these timelines were articulated with the academic narrative biographies. The use of colors, symbols according to the event, and quotes from the interviews in each of the events within the biographical lines, allow us to realize that, in the case of men, work trajectory follows a progressive linearity and it distanced from the trajectory family. On the contrary, for women work trajectory is put into evaluation and stressed regarding the family trajectory. Thus, we concluded that the visualization of the timelines is a flexible tool because it can act as a memory aid to the interviewees during fieldwork. Besides, it allows to account for subjective aspects to be analyzed, to make comparisons, and to show the information amiably for the audience and research groups.

KEYWORDS Data visualization; biographical approach; qualitative methodology; academic trajectories; gender comparison; subjectivity. 


\section{Introducción}

En general son los estudios cuantitativos los que utilizan la visualización de datos para clarificar, describir, y profundizar los resultados obtenidos. En el caso de este artículo, queremos proponer que la visualización de datos también puede utilizarse en estudios cualitativos. Sostenemos que el uso de visualización de las líneas de tiempo en lo cualitativo equivaldría al rol que cumplen las tablas y gráficos en los datos cuantitativos. Para demostrar esto, trabajamos sobre las diferencias de género dentro el ámbito laboral y familiar, respecto de cuatro trayectorias de académicos/ as chilenos/as de un departamento de química. A partir de estos casos de estudio se pretende dar cuenta de que por medio de la visualización de las líneas de tiempo de los/as académicos/as chilenos/as, se puede informar de manera más amigable y simple la diferenciación según el género. Así, este artículo demuestra la utilidad del uso de líneas de tiempo como complemento visual de las narrativas, contribuyendo a la discusión sobre los usos de los estudios biográficos.

En general, los estudios sobre trayectorias académicas, se han focalizado en el ámbito laboral. Sin embargo, en este artículo nos hemos propuesto trabajar con un concepto de trayectoria académica basándonos en la idea de Coninck y Godard (1998), sobre las trayectorias como una imbricación de varias historias de diferentes ámbitos de la vida. De esta forma, se propone una conceptualización de las trayectorias académicas, teniendo en cuenta las dimensiones familiares, personales y de pareja. La utilización de este concepto como punto de partida teórico parece necesaria en un campo de estudio donde la trayectoria familiar no suele ser incorporada al definir el concepto de las trayectorias académicas (Berríos, 2005; Martínez, 2012). Cuestión que parece implausible si es que adherimos a la idea de Coninck y Godard. En otras palabras, en este artículo las trayectorias académicas serán entendidas como:

"las narraciones (secuencias de eventos, experiencias y significaciones, entre otros) relacionadas con sus vidas familiares, personales y de pareja (cruzados por el género y la nacionalidad); desarrolladas en un espacio llamado la academia (inserto generalmente en la institución universitaria), en que se juegan estrategias de competición y de cooperación. Así se releva que las trayectorias académicas no sólo son lo que concierne al contexto universitario o institucional, sino que también es necesaria la consideración de la vida familiar, personal y de pareja, en tanto producen y coproducen variadas tensiones, sinergias y retroalimentaciones entre todos estos cuatro ámbitos" (Ortiz, 2017, pp. 270-271).

Usualmente las investigaciones se han centrado en estudiar una sola cara de la trayectoria, pero no la imbricación de familia y academia (Ortiz, 2017), que es lo que aquí utilizamos a modo de ejemplo del uso de la visualización de datos cualitativos. 
De esta forma, el artículo está organizado de la siguiente manera: en primer lugar, se muestran algunos antecedentes relevantes sobre desigualdad de género en el ámbito académico y laboral, luego se desarrollan los aspectos teóricos sobre visualización de datos; en tercer lugar, se presenta la metodología de la investigación; en cuarto lugar, se da cuenta de los resultados por medio de la comparación de las trayectorias familiares y laborales de cuatro académicos/as; y, finalmente, se explicitan conclusiones de síntesis y limitaciones de la visualización.

\section{Antecedentes: desigualdad de género y academia}

Debido a la relevancia que tiene la noción de "género" en este artículo se menciona su definición desde la perspectiva de las autoras. Este estudio se adscribe a la siguiente noción, propuesta por Simbürger y Undurraga: "la construcción social basada en creencias, expectativas, valores, actitudes, comportamientos, roles y lo que se espera que sean y parezcan hombres y mujeres en una sociedad y en un tiempo determinado. El género no es estático ni determinado al nacer, sino que es dinámico a lo largo de la vida y es relacional. Por lo tanto, género es una categoría sociológica, así como otras como clase social, raza/etnia, etc." (Simbürger y Undurraga, 2013, p. 171).

La postergación de la maternidad a favor de tener mayor independencia económica, o logros profesionales (Montilva, 2008), se relaciona con "el desarrollo de una biografía a través del mercado laboral y académico" (p. 79). En este sentido, es el nivel socioeconómico y el grado de escolaridad variables que influenciarán las posibilidades de tener mejores oportunidades laborales, los cuales tendrán a su vez un impacto fundamental en la postergación o no de la maternidad (Fuentes et al., 2010). Pues, muchas veces para acceder a mejores puestos laborales, tener hijos/as es visto como un impedimento para el desarrollo progresivo de las carreras profesionales de las mujeres. En función de esta premisa, es que precisamente se funda este artículo, pues consideramos que la biografía se desarrolla en la competencia de roles entre familia y trabajo (Elder, 1985). En este sentido, existe una batería de teoría respecto de las trayectorias académicas y las diferencias de género.

En Chile, por ejemplo, según Ramos (2012), "el campo (de las ciencias sociales chilenas) presenta claros clivajes marcados por el género" (p. 38). Estas diferencias se manifiestan desde el comienzo de la carrera científica, en el acceso, la participación (Bernasconi, 2010; Stefoni, 2010) y durante su desarrollo (Mora, 2010; Sonnert \& Holton, 1996). En el caso de las trayectorias académicas femeninas, existe, por un lado, evidencia sobre el éxito de las trayectorias académicas de mujeres que rechazan o posponen el matrimonio y la tenencia de hijos (Kyvik, 1990; Mason \& Goulden, 2004). Y por otro, existen estudios donde los datos no presentan asociación significativa entre matrimonio, tenencia de hijos/as y las trayectorias académicas, cómo menciona Cole \& Zuckerman (1987) (estudios de carácter transversal y no longitudinal). 
Existiendo evidencia en ambos sentidos, lo que proponemos en este artículo, es más bien indagar en estas trayectorias, constituyentes de la biografía, las significaciones y valoraciones de las decisiones que los sujetos van tomando en su vida.

Por otro lado, en términos generales, la comparación de las distintas trayectorias académicas desde una perspectiva de género ha sido estudiada con un enfoque transversal y concentrándose sólo en la visión de las mujeres (Cerros \& Ramos, 2011; Chinchilla, León, Torres \& Canela, 2006). Pues, regularmente son las mujeres quienes se focalizan en carreras más devaluadas en salarios y prestigio que los hombres (England \& Farkas, 1986; England, 2010), lo que implicaría mayores desigualdades en sus trayectorias. Esta situación de desigualdad se mantiene a lo largo de la trayectoria laboral (Berríos, 2005; Martínez, 2012), ya que como describe Mauro (2004), son las mujeres las que están dispuestas a ganar menos y ocupar puestos de menor prestigio y poder, si es que eso implica que puedan tener más tiempo para la familia. Las mujeres, señala la autora, están a lo largo de sus vidas en una constante búsqueda de equilibrio entre familia y trabajo, lo que las deja en desventaja respecto de sus compañeros en el momento de acceder a capacitaciones y promociones laborales. Desde la visión de los altos cargos, esto mismo es visto de forma negativa para la productividad de la empresa, ya que demostraría que ellas son las que están constantemente subordinadas a los ciclos de la vida familiar (Mauro, 2004). Así, la identidad de las mujeres vinculadas a la realización personal mediante la dimensión profesional entraría en conflicto con las expectativas sociales de ser "buenas madres y parejas" (Cangiani \& Montes, 2010, p. 197). En el mismo sentido, Martínez (2012) postula que, los estudios sobre trayectorias académicas al focalizarse sólo en los espacios universitarios (Lavados, 2006) -debido a que este es por excelencia el lugar de desarrollo de los/as académicos/as (Gilbert, 2011; Ramos, 2012) - , para el caso de las mujeres invisibilizaría la disputa y tensión existentes entre identidades, pues la "tensión entre lo laboral y lo familiar "tiene que ver sobre todo con los roles y los tiempos, [las cuales] muchas veces (...) son antagónicos entre sí" (Martínez, 2012, p. 154). Sin embargo, la autora Knorr Cetina (2005) resalta la consideración del ámbito familiar para la fabricación de una trayectoria académica. De esta forma, si es que nos proponemos estudiar las trayectorias académicas es imperativo considerarlas. Más allá de las consecuencias objetivas que las formaciones familiares y las relaciones amorosas puedan tener en el desarrollo de una trayectoria laboral, lo que nos interesa es mostrar, por medio de la visualización de líneas de tiempo, cómo los sujetos, durante el periodo de formación familiar y los periodos de unión o desunión de una pareja, significan estas etapas. Desde esta perspectiva, el enfoque biográfico y su visualización se hacen útiles pues promueven la relevancia de las significaciones que los sujetos les dan a sus experiencias.

Lo que proponemos en este artículo es poder mostrar la utilidad de la visualización de trayectorias por medio del contraste de las diferentes trayectorias académi- 
cas, desde una dimensión visual y narrativa, visibilizando las diferencias existentes entre hombres y mujeres en la carrera académica. Demostrar esto hará un aporte respecto del desarrollo de entrevistas biográficas y de presentación de resultados que es el foco de este artículo. Creemos que, aunque el objetivo general del artículo es la visualización de datos; también se logra aportar al estudio de género y trayectorias académicas, a pesar de ser usado a modo de ejemplo para dar cuenta de la utilidad práctica de la visualización de trayectorias. Por este motivo, se consideró al enfoque biográfico (Bernasconi, 2011; Cornejo, 2006; Cornejo, Kornblit, 2004; Mendoza \& Rojas, 2008; Moyano \& Ortiz, 2016, 2018), y la perspectiva de género (Longo, 2009; Selva, Sahagún \& Pallarès, 2011) como metodología de investigación. Así, se propone complementar el enfoque biográfico con la visualización de trayectorias de vida durante la recolección, análisis y presentación de datos. Pues, las líneas de tiempo tienen la capacidad de organizar los datos y hacer más accesible y sintética la información, por tanto, mucho más fácil de procesar y favorecer a la interpretación (Hiller, 2011).

\section{Enfoque teórico}

Como se mencionó en la introducción, el objetivo principal de este artículo es dar cuenta, por medio de la visualización de las trayectorias, la utilidad metodológica de la visualización de datos. En función de este mismo objetivo, presentamos las bases sobre las cuales se funda la relevancia de la visualización de datos cualitativos y, específicamente, de las líneas de tiempo.

\section{Visualización de datos cualitativos}

La visualización de datos permite sobrepasar las capacidades humanas, superando las limitaciones de la cognición y memoria (Munzner, 2014). Así los datos, a través de la visualización, se transforman a escala humana permitiendo una mayor comprensión de la información (Manovich, 2008). La visualización tiene como objetivo primordial revelar de forma simple la información por muy compleja que sea. De esta forma, las visualizaciones no solo transmiten información de manera estética, sino que una buena visualización es aquella que entrega información de manera eficiente y logra generar nuevo conocimiento (Tabin, Shaugat Abdullah, Ahmed \& Giunchiglia, 2013). Es así como en este caso se entenderá visualización como la representación visual de conjuntos de datos (Munzner, 2014) que permiten la exploración, el análisis y el estudio de estos (Cairo, 2011).

En la era de los datos, la visualización, disciplinar que actualmente se ha consolidado como un área académica, se ha encargado de dar forma mayoritariamente a datos cuantitativos, puesto que visualizar datos cualitativos no es una tarea fácil. Los datos cualitativos se desprenden de preguntas tales como ¿Por qué? y ¿Cómo?, las cuales son difíciles de convertir en imágenes. De hecho, muchas veces se tiende 
erróneamente a cuantificar este tipo de datos en las visualizaciones, perdiendo su intrínseca riqueza. Sumado a esto, actualmente las técnicas de visualización de datos cualitativos parecen ser bastante limitadas (Henderson \& Segal, 2013).

Henderson y Segal (2013) realizaron una categorización de los tipos más comunes de visualizaciones cualitativas, como la nube de palabras, análisis de sentimiento, red de frases, entre otros. También destacan los gráficos cronológicos, lo que acá nombramos: líneas de tiempo.

\section{Visualización cronológica y línea de tiempo}

El tiempo como variable, introducido en las distintas formas de visualización de datos, ha sido fundamental para el desarrollo de diversos gráficos y ha sido foco de atención de esta disciplina. Tufte (2001) plantea que con esta sola dimensión, que puede ser medida en distintas escalas, la visualización adquiere eficiencia y fortalezas para la interpretación.

Una línea de tiempo es una herramienta de visualización de información que se utiliza para comunicar una secuencia de eventos relacionados. La línea de tiempo parece ser común a muchas culturas y distintos tiempos, sin embargo como la conocemos actualmente, la línea de tiempo moderna parece tener pocos siglos (Coulson \& Pagán, 2014). Esta surge en la era moderna como una concretización gráfica de la historia en sí misma (Rosenberg \& Grafton, 2010). Lo mismo sucede con los participantes de este estudio, es su propia biografía la que aparece materializada en la visualización.

Joseph Priestley es quien propone la forma moderna de la línea de tiempo. Una de sus obras más conocidas es "The New Chart of History" (1769), donde muestran los destinos de setenta y ocho reinos e imperios durante un periodo particular. La contribución que hace Priestley es que regulariza la distribución de las fechas y la orientación horizontal (Rosenberg \& Grafton, 2010).

Como plantean Rosenberg \& Grafton (2010) el credo de la vida moderna requiere esfuerzos importantes para manejar gran cantidad de información, es acá donde la visualización de datos tiene un papel fundamental, pues para manejar y comprender esa gran cantidad de información es necesario traspasar la barrera cognitiva humana a través de las herramientas de la visualización. Esto no es ajeno a las líneas de tiempo, ya que se requiere contar historias de muchos años en un pedazo de papel, lo que demanda esfuerzos de economía visual, para que la información se pueda visualizar de manera efectiva, eficiente y coherente. En el caso de las líneas de tiempo de las historias de vida, sucede lo mismo, se cuenta una historia, en la que se deben plasmar experiencias, eventos, puntos de inflexión, etc. En definitiva, se visualiza complejidad. 
En el caso específico de las líneas de tiempo del presente estudio, la visualización facilita la comparación entre las trayectorias académicas y cómo se han configurado en función de su género. A pesar de las facilidades y beneficios que puede tener visualizar la vida de un individuo, siempre se debe trabajar con bastante cautela para no sobre simplificar la información. Así lo reafirma Hiller (2011): “(...) los gráficos no deberían sustituir las aproximaciones analíticas para sacar conclusiones respecto de los significados que los individuos le atribuyen a los hechos de su vida" (p. 1030, traducción de las autoras). Junto a otras formas de análisis del enfoque biográfico, las líneas de tiempo son un aporte a la visualización de datos cualitativos a pesar de que frecuentemente se encuentran subrepresentadas y poco exploradas.

\section{Metodología}

Este artículo utiliza cómo fuente de material empírico, entrevistas realizadas durante el año 2014. Las cuales tenían por objetivo conocer la relación entre las trayectorias académicas y las vidas familiares en la academia universitaria chilena, considerando desigualdades de género. Se seleccionó el caso de los/as académicos/as de un departamento de química en una universidad chilena. Siguiendo la recomendación de Cornejo et al. (2008) se entrevistaron a 17 académicos/as (9 hombres, 8 mujeres ${ }^{1}$; de los cuales 6 eran académicos asistentes, 6 eran asociados y 5 titulares), para garantizar la profundización de los análisis con los recursos existentes. El artículo presenta solo cuatro casos para dar cuenta de la utilidad de la visualización de datos, es decir plantea una reflexión metodológica respecto de las trayectorias de vida. Los casos específicos y la universidad a la que pertenecían se mantendrán en el anonimato para respetar la confidencialidad de los datos. La elección de la muestra de los/as entrevistados/ as se realizó según el sexo y el tipo de cargo que desempeñaba en el momento de la entrevista. Por cada caso se hicieron tres encuentros, que duraron aproximadamente una hora, y fueron grabados, transcritos y codificados entre los meses de agosto del 2014 y enero del año 2015. La metodología fue de índole cualitativa lo cual permitió aprehender la subjetividad de los/as entrevistados/as (Taylor \& Bogdan, 2000), por medio del estudio de caso múltiple (Stake, 2013).

El concepto de subjetividad aquí utilizado, ha sido ampliamente trabajado por las autoras Moyano y Ortiz (2018), y específicamente en su interrelación con lo que es el enfoque biográfico (Moyano y Ortiz, 2016). En breve se puede decir que es el aspecto más personal del sujeto, es su interioridad, pensamiento, significaciones, valoraciones y emociones que se ven entrelazadas con las experiencias y eventos que le van ocurriendo en su vida. El aspecto subjetivo es esencial para investigaciones que usan el

1. La reducción del número se debe principalmente a la baja presencia de mujeres titulares, sumado al escaso tiempo de parte de ellas, presentando mayores dificultades de acceso. 
enfoque biográfico, debido a su impacto a largo plazo en la vida de las personas. Así, dado que el estudio de las subjetividades permite problematizar los que los sujetos experiencian cómo intérpretes de estas mismas narrativas (Bernasconi, 2011), es que radica la importancia de esta noción relevando su origen del vínculo mismo entre el individuo y la estructura.

Dada la relevancia que adquiere el relato sobre la historia laboral y la familiar de los/as entrevistados/as, es que se optó por el método biográfico (Bertaux, 1989; Ferrarotti, 2011; Guerra \& Skewes, 1999; Kornblit, 2004; Pujadas, 2002; Sautu, 2004). Este método permitió la recaudación de información con un mayor nivel de detalle sobre episodios vividos, junto a las emocionalidades asociadas a esos episodios (Bernasconi, 2011; Sharim, 2005). En específico se realizaron narraciones biográficas acotadas al objetivo de estudio. El procesamiento de la información se realizó mediante el análisis comprensivo de Bertaux (1981; Kornblit, 2004). Se puso especial énfasis en lo(s) punto(s) de viraje que le otorga sentido a las continuidades y las discontinuidades en las trayectorias, identificando desigualdades de género en las carreras laborales (Arango \& Viveros, 1996; González, 2010). Se usó el programa MAXQDA 11 para la posterior codificación y el análisis de los datos.

Para profundizar en la utilidad de líneas de tiempo cómo una herramienta de investigación y de presentación de datos longitudinales, como ya se ha dicho, nos concentramos en este artículo en particular en el su uso en cuatro casos de este estudio. Estos casos fueron elegidos pues ilustran de mejor forma la particularidad del uso de la visualización de las historias de vida en las líneas de tiempo que proponemos.

Si bien los relatos biográficos de cada uno de los entrevistados son singulares en sus aspectos temporales, en función del análisis longitudinal comparativo, se requiere ordenar cronológicamente las trayectorias. Se utilizaron las líneas biográficas durante su trabajo de campo de forma sencilla: en la medida que se revisaban las entrevistas transcritas, se dibujaban en una hoja los eventos que marcaron la trayectoria académica y la vida familiar, presentados de forma cronológica (identificando el año y a veces el mes). Y, se le agregó una frase breve que describiera lo que había significado cada evento para el/la entrevistado/a. Luego, eran discutidas con los/as entrevistados/as en las siguientes sesiones. Fue una propuesta metodológica que le ayudó a los/ as entrevistados/as a recordar algunos eventos después de la primera reunión, ayudó a la codificación y análisis posterior de la entrevista, y a presentar la información adecuadamente. Para su construcción se usaron sólo las entrevistas como fuente de información, pues, en general, en el enfoque biográfico los datos son las narraciones de la vida, considerando que su veracidad radica en lo que los/as entrevistados/as relatan (Moyano \& Ortiz, 2016). El relato libre de los/as entrevistados permitió la estructuración de las líneas como se les presentaron a ellos/as. Lo cual, es un primer aporte de la visualización de datos cualitativos. 
Durante la aplicación de las entrevistas se descartó la coproducción de las líneas de tiempo pues entregar herramientas cronológicas a los entrevistados a priori podría producir narrativas estandarizadas opacando el uso del tiempo subjetivo por parte de los narradores (Moyano \& Ortiz, 2016). Las líneas sólo se utilizaron como "ayuda memoria" para darle continuidad a la conversación. Esta "ayuda memoria" se basa en la asociación que los sujetos realizan de sus propias historias de vida. En cuanto a la posibilidad de contar con lagunas de memorias, el hecho de haber entrevistado a personas de entre 25 y 65 años, que no tenían algún tipo de dificultad, redujo enormemente esta posibilidad. Luego, la sistematización de las trayectorias académicas (visualización y narrativa) se focalizó en las diferencias entre los/as entrevistados/as respecto de las decisiones de parentalidad y uniones o desuniones amorosas/maritales.

La visualización se diseñó diferenciando con dos colores las trayectorias laborales (celeste) y familiares (naranjo), además, se agregaron íconos sobre la temática del evento, y una cita de la entrevista. Es importante destacar que las líneas no sustituyen el relato abierto de los sujetos, por lo que se recomienda reforzar la intención de que sean los propios entrevistados quienes construyen su propia cronología, y el entrevistador oriente hacia el objetivo de investigación. Los resultados que se muestran a continuación dan cuenta de la imbricación entre trayectorias familiares y laborales (Coninck \& Godard, 1998) realizada por los entrevistados/as. Estas trayectorias se componen de la asociación de algunos de los eventos de ambas trayectorias, pues en términos empíricos es así como los sujetos narran su vida.

\section{Resultados: Las trayectorias académicas y familiares de científicos/as. Organiza- ción de los relatos mediante las líneas biográficas de tiempo}

A modo de ejercicio práctico, presentamos a continuación cuatro casos, la descripción de sus líneas de tiempo, la visualización y un análisis final de cada historia focalizado en la utilidad que tiene la visualización para dar cuenta de aspectos analíticos de las trayectorias.

Agustín: El quiebre no desestabiliza mi objetivo².

Profesor asociado, 46 años, separado con trillizos adolescentes y actualmente sin una relación de pareja.

Al comienzo de la línea se puede observar que Agustín ingresa a estudiar su pregrado (1988), para luego continuar con el doctorado (1993), ya que "nos pedian tener el doctorado [y al titularse ya] sabías que ibas a seguir una carrera académica" (Agustín, 11 de agosto del 2014, Chile).

2. Estos subtítulos que asignamos a cada historia son frases asignadas por las autoras que resumen, narrativamente, la historia de cada participante. 
Entre los años de ingreso al programa doctoral y de inserción en la academia, tuvo una relación de pareja que recuerda con nostalgia y emoción (distinguida en el año 1993). Él menciona que ella lo apoyaba mucho con sus estudios. En esa misma época, nace una gran preocupación por temas económicos pues, las becas de doctorado no alcanzaban para sustentar a dos personas. De hecho, esta fue una de las razones por las cuales esta relación terminó. Más tarde, ya habiéndose incorporado en la universidad (1999), se casa con una nueva pareja, nacen sus tres hijos, y obtiene el grado de doctor. Agustín les da mucha importancia a esos años (1999-200o) y lo constituye como un hito en su biografía a partir de varias dimensiones. En primer lugar, por tener a los trillizos, pues a pesar de que no fue algo planificado, tampoco fue no deseado; más bien lo significa como una gran alegría en su vida. Y en segundo lugar, el éxito con la acumulación de proyectos Fondecyt ${ }^{3}$, lo cual le permite su mantención en la academia, proyectos que resultan ser la "unidad de medida que se privilegia (...) si no tienes un Fondecyt, tu pie cuelga" (Agustín, 11 de agosto del 2014, Chile).

Como se puede ver en al final de su línea, Agustín siguió publicando, asistiendo a conferencias e impartiendo los mismos cursos. Seis años después obtiene una plaza postdoctoral en el extranjero (2006). Se va de Chile sin su familia, ya que al reconocer que la academia es un espacio que "nunca han tenido horarios" (Agustín, 11 de agosto del 2014, Chile), era mejor priorizar que sus hijos siguieran viviendo en Chile. Los niños entonces se quedan con su madre, a pesar de que la universidad le entregaba varias facilidades para mudarse con su familia completa. Al finalizar su contrato, y al volver al país, Agustín se separa de su esposa y sus tres hijos continúan viviendo con ella (2007, último evento de la línea). Las líneas de tiempo permiten, como se ve en el caso de Agustín, seguir la descripción de su trayectoria, de manera mucho más simple, y por tanto comprensible. Seguir la lectura a la luz de la gráfica permite identificar con años y símbolos el entrelazamiento de cada hito. De esta forma la visualización como complemento de las narrativas nos permiten observar cómo, en la historia de Agustín, la paternidad es un hito importante, pero no logra desencadenar acomodaciones en su carrera académica. Se puede visualizar a simple vista cómo los eventos están distribuidos en las líneas laborales y familiares de manera bastante homogénea. Los quiebres y distancias familiares, no repercuten en su vida laboral que más bien sigue una linealidad y un orden progresivo. Agustín no desvía su trayectoria laboral y sus objetivos respecto de esta.

3. Fondo Nacional de Desarrollo Científico y Tecnológico de Chile. 
"POR MÁS QUE YO TRABAJE, TRATO DE ACOMODAR TODO A MI HIJO": USOS DE LA VISUALIZACIÓN DE LÍNEAS DE TIEMPO PARA COMPRENDER DESIGUALDADES DE GÉNERO EN LA ACADEMIA

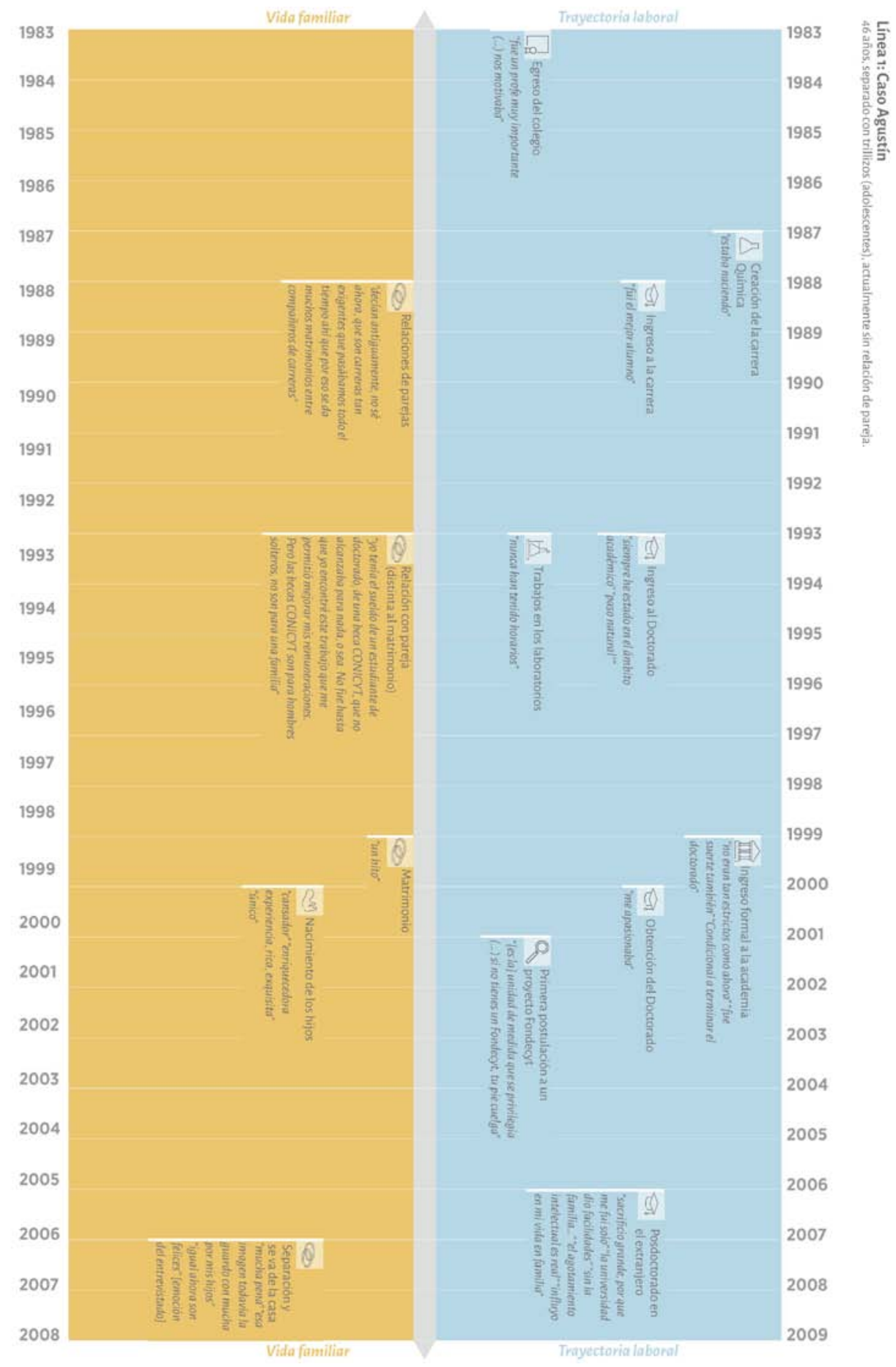


Ignacia: "ya es tarde para mi".

Académica titular de la Facultad de Química, 61 años, sin relación de pareja actual, sin hijos biológicos.

Ignacia vivió en México 11 años antes instalarse nuevamente en Chile (1971-1980), donde estudió el pregrado en Química y trabajó "ad honorem" durante el mismo periodo. Luego, la contrataron formalmente como académica en la misma universidad (1979), y volvió a Chile el año 1980 para poder estar cerca de su sobrina. Pero en su retorno, comenta, que todo "fue muy duro" (Ignacia, 18 de agosto del 2014, Chile) pues comenzó su trayectoria laboral nuevamente. Ignacia, desde ese año, trabajó en todos los proyectos que surgieran.

Años después, realiza sus estudios para la obtención del doctorado (1992-1998). Durante el segundo año de sus estudios, la contratan formalmente en la universidad como instructora. Durante el doctorado, Ignacia no dejó nunca de trabajar (destacado en 1992 en la línea). Era imperativo trabajar pues no siempre obtuvo financiamiento. En este escenario (el del doctorado) fue siempre postergando la maternidad. Hoy, declara, es "muy tarde para mi" (Ignacia, 18 de agosto del 2014, Chile) (destacado en 1992). No obstante, ha resignificado la maternidad (destacado en 1998) por medio del vínculo importante con su sobrina.

La vida personal y familiar de Ignacia se vio reorientada por sus estudios y su carrera académica hacia trayectorias que ella no planificó, sin embargo, sus estudios también se vieron afectados y retrasados por las circunstancias históricas y familiares que le tocaron vivir. Ignacia tuvo que irse del país a causa de la dictadura militar ${ }^{4}$ que en Chile se vivía. Recuerda (alude al año 1973 en la línea) que "fue muy duro... fracturó a la familia" (Ignacia, 18 de agosto del 2014, Chile) porque todo estaba en distintos lugares del mundo, y ella además disponía de muy poco tiempo para comunicarse con todos.

La narración de Ignacia fue poco cronológica, de hecho, termina con el primer evento de su línea (el exilio producto de la dictadura). En este sentido, la visualización aporta en el orden y comprensión descriptiva de su trayectoria de vida.

4. La dictadura militar en Chile tuvo una duración de 17 años, desde 1973 hasta 1990. 
"POR MÁS QUE YO TRABAJE, TRATO DE ACOMODAR TODO A MI HIJO": USOS DE LA VISUALIZACIÓN DE LÍNEAS DE TIEMPO PARA COMPRENDER DESIGUALDADES DE GÉNERO EN LA ACADEMIA

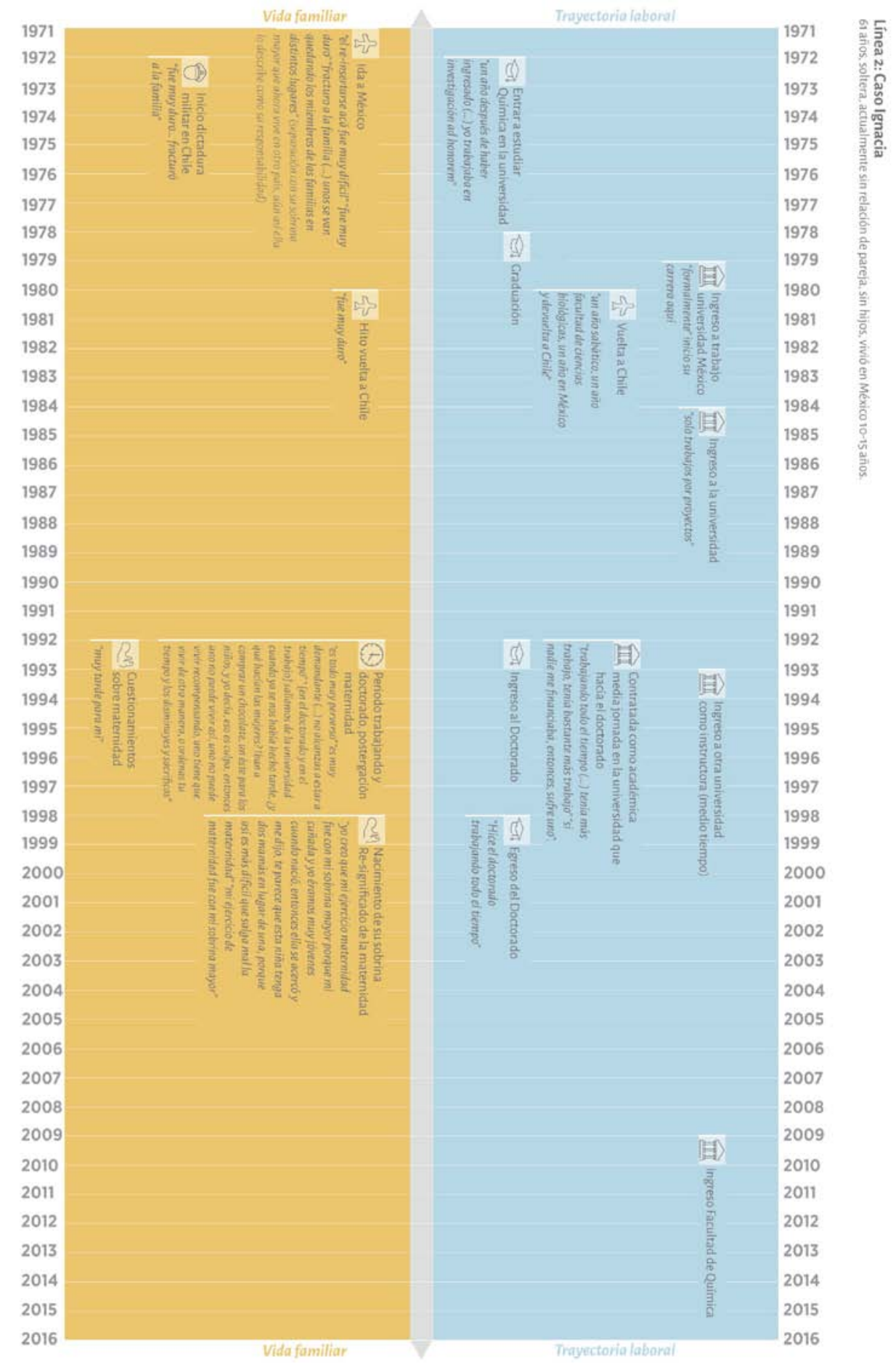


El retorno a Chile desde México producto de relaciones familiares y una maternidad elegida en relación con su sobrina, modifican el rumbo y el desarrollo de su trayectoria académica, pero, sobre todo, generan una reflexión evaluativa de parte de ella sobre las implicancias actuales de la no-maternidad, como una decisión realizada en el pasado respecto de anteponer su trayectoria académica a su vida personal. La línea nos permite visualizar cómo el periodo de decisión de postergación de la maternidad se realiza durante los mismos años en los que Ignacia comienza su carrera académica, y obtiene lo que será su plaza de trabajo durante varios años. A partir de esto, es claro que las decisiones de no-maternidad guardan estrecha relación con lo que en su dimensión laboral estaba viviendo. De hecho, vincula su carga de trabajo con la poca atención a su vida personal.

Ignacia tiene un sentimiento de ambivalencia respecto de su maternidad, entrelazando conformismo y frustración en su relato, cuando asegura que si no hubiese realizado sus estudios de postgrado e ingresado a la vida académica con contrato tan tardíamente (2009), hubiese sido madre. Sin embargo, no siente arrepentimiento, pues su sobrina cumplió y cumple un rol fundamental en función de esos deseos.

Francisco: el trabajo, mi dedicación exclusiva.

Profesor asociado de 54 años, es de origen argentino. Decidió no tener hijos, y está casado con una mujer también argentina.

En el comienzo de la línea se da cuenta de que Francisco egresó de su etapa escolar con la especialidad de batidor y técnico en química. En 1984 ingresa a la universidad, pero como no le gustó decide trabajar fuera de la universidad durante esos años (1984-1985): "Por eso me aparté de la universidad y me fui a trabajar" (Francisco, 11 de agosto del 2014, Chile). En 1985 contrae su primer matrimonio y un año después migra, por razones laborales, desde Argentina (su país natal) a vivir a Brasil durante un año. Después de su regreso a Argentina ingresa a estudiar a la universidad química e ingeniería química. Trabajó en Argentina como micro empresario y se separa de su primer matrimonio.

En 1992 se casa por segunda vez y toma la decisión junto a su pareja de no tener hijos, debido a que: "Es una cuestión, digamos, dentro del contexto en que nosotros nos movíamos, no teníamos planes de estar mucho tiempo en un lugar asentados". (Francisco, 11 de agosto del 2014, Chile). En 1994 termina su doctorado en química y se muda a Estados Unidos por un post doctorado. Luego de esto comienza a trabajar en Chile. Buscó trabajos en distintos lugares del mundo, pues en Argentina no tuvo muchas posibilidades. Finalmente encuentra trabajo en Chile. 
"POR MÁS QUE YO TRABAJE, TRATO DE ACOMODAR TODO A MI HIJO": USOS DE LA VISUALIZACIÓN DE LÍNEAS DE TIEMPO PARA COMPRENDER DESIGUALDADES DE GÉNERO EN LA ACADEMIA

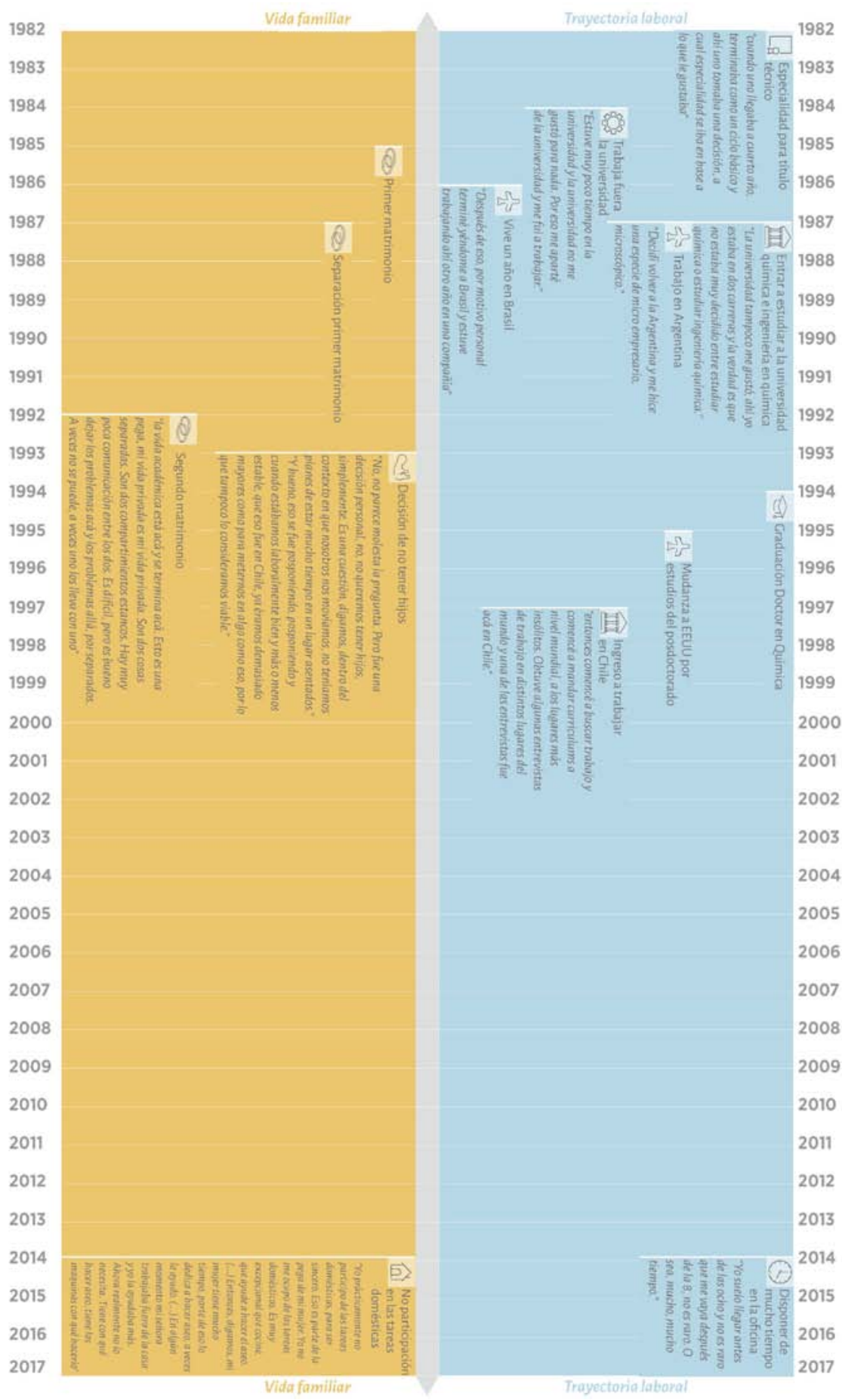


Francisco confiesa abiertamente que él no participa en las tareas domésticas. Lo justifica recalcando que él tiene una alta carga laboral, "Yo suelo llegar antes de las ocho y no es raro que me vaya después de las ocho, no es raro. O sea, mucho, mucho tiempo (en el trabajo)" (Francisco, 11 de agosto del 2014, Chile). En cambio, comenta Francisco, su mujer no trabaja fuera de la casa y es la encargada de las tareas domésticas, de hecho reafirma que "ella realmente no lo necesita" (Francisco, 11 de agosto del 2014, Chile) (su ayuda).

En el caso de Francisco, es clave la disminución -bordeando la ausencia- de eventos desde 1997 (lo que puede observarse fácilmente en la visualización), pues esto podría interpretarse como estabilidad respecto de ambas líneas. Sin embargo, destaca al final de su línea (año de realización de las entrevistas) dos transiciones, que refieren a la prioridad de su trabajo respecto de su vida familiar, pero sobre todo de la vida doméstica. Por otro lado, al igual que Ignacia, Francisco decidió no tener hijos, sin embargo y a diferencia de ella, en la lectura de las citas y de las narraciones se puede observar una decisión sin mayores tensiones "decidimos no tenerlos, simplemente" (Francisco, 11 de agosto del 2014, Chile).

\section{Trinidad: La responsable principal}

Profesora asistente de 42 años, con dos hijos, casada con un hombre chileno. De origen brasileño, decidió tener hijos biológicos mientras estaba en una posición postdoctoral.

El año 1995 Trinidad se gradúa de la carrera Farmacéutica, el año 1998 termina su magister, y el 2005 el doctorado. Desde el año 1998 realiza trabajos independientes como profesora, y participa en varias investigaciones, hasta el año 2006. Ese año viaja a Chile a realizar una pasantía, y vuelve el año 2008 por un postdoctorado en la misma universidad, pues ya conocía a algunos profesionales que trabajaban los temas que le interesaban. Trinidad comenta que sus primeros años en Chile tuvieron mucha relevancia en su vida, pues durante la pasantía conoce a quien es hasta hoy su marido. Es debido a esta relación que ella decide volver a Chile (destacado en año 2007 en línea).

Trinidad tuvo que comenzar su trayectoria académica prácticamente de nuevo. En Brasil, había construido extensas redes de colaboración científica, trabajaba en distintos proyectos y tenía una trayectoria docente consolidada. La migración se distingue como un elemento central en su trayectoria académica, pero también en la trayectoria familiar. Sobre todo al hablar de la maternidad, "es un cambio de vida súper heavy (...) también fue muy difícil. Porque igual tuvo un hijo fuera del país, tuve un hijo con todo un mundo muy distinto del que vivía yo, era una realidad que yo no conocía" (Trinidad, 15 de octubre del 2014, Chile). 
En sus primeros años contratada en la universidad (2009), dejaba a su hijo en una guardería que, según ella, funcionaba bien. Sin embargo, era ella la responsable de los traslados pues, su horario era más flexible que el de su marido. De igual forma, contrata a una persona externa como soporte en las tareas domésticas y de cuidado. Esta ayuda para Trinidad resulta fundamental, e incluso comenta que no hubiera podido continuar su trayectoria académica de no haber contado con la opción de pagarle a alguien para realizar estas labores y la relación de confianza que ambas han logrado construir.

Toda la carrera académica, cambió radicalmente luego de su decisión de vivir en Chile para formar una familia. El tener hijos para ella fue un gran cambio, pero redujo casi a cero sus tiempos de pre y postnatal en función de continuar lo más establemente posible en su trayectoria académica: "di clases todo mi embarazo, hasta los 8 meses estuve acá dando clases" (Trinidad, 15 de octubre del 2014, Chile), "Lo que tuve que hacer fue que yo trabajé embarazada, y al mismo tiempo yo estaba haciendo mi postdoc, y es que arreglamos un poco el proyecto para que yo siguiera trabajando sin muchas sustancias tóxicas" (Trinidad, 15 de octubre del 2014, Chile).

En el caso de Trinidad, su vida cambió por completo luego de casarse y tener hijos, pues aquello no solo significó cambiarse de país, si no también modificar todas sus redes y los avances en su trayectoria académica desarrollada en Brasil. Trinidad al contrario de Agustín, cambió una vida académica estable en Brasil por venir a Chile donde vive su actual marido.

Ella comenta que, al vivir lejos de su familia de origen, no contó con la ayuda de ningún familiar cercano para tareas de cuidado (aun cuando su marido es chileno y la familia de él también). Trinidad destaca en todo momento que la decisión de ser madre fue sobre todo eso, una decisión, sin embargo, los sacrificios que destaca los realizó principalmente en función de no "desaparecer" de su contexto académico. A pesar de ello, describe a la maternidad como "(lo) más importante para una mujer, por más que uno trabaje, tenga mil otras cosas, creo que cuando uno tiene hijos y chicos, su vida acaba amoldándose a su hijo. Por lo menos la mía es así. Por más que yo trabaje, yo trato de acomodar todo a mi hijo. Pero el hecho de ser madre es único, yo creo" (Trinidad, 15 de octubre del 2014, Chile).

Es interesante cómo las trayectorias visualizadas en los casos de Trinidad e Ignacia entregan información similar. Existe en ambos casos una acumulación visual de eventos en las líneas laborales y familiares, pero con sentidos distintos para cada una. En el caso de Trinidad, es la movilidad geográfica y el nacimiento de sus hijos los que coinciden con estos eventos, y no la decisión de no ser madre como en el caso de Ignacia, sin embargo, y al igual que en el caso de Ignacia ambas líneas laboral y familiar están en tensión. En la narración y en las citas de los eventos se pueden observar como los eventos respecto de lo laboral tienen aspectos de la línea familiar y viceversa. 


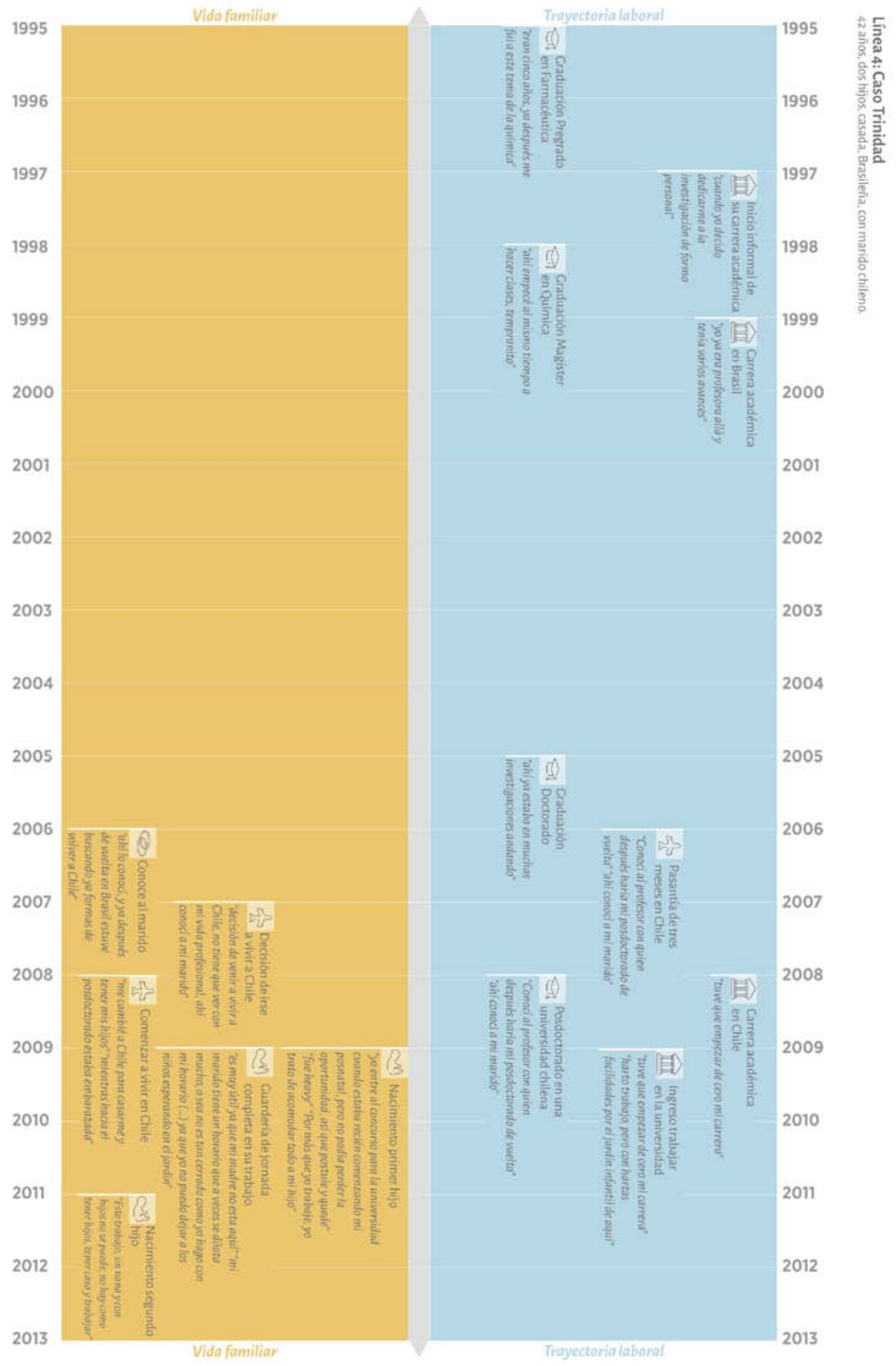


Es posible visualizar las diferencias de género entre los tres casos de Agustín, Trinidad e Ignacia, al concentrar el análisis en como enfrentan la maternidad y en qué medida las trayectorias de vida completas se construyen a partir de esos hechos es notoria. En el caso de los hombres, tal como vimos en el de Agustín, la paternidad es un hito importante y central, pero no logra desencadenar una serie de cambios o acomodaciones por parte de él en su carrera académica. De hecho como vimos en la línea de tiempo, esta continúa su línea sin sufrir muchas modificaciones. Mientras que en el caso de Trinidad, sucede todo lo contrario, su vida cambió por completo luego de querer casarse y tener hijos con su pareja, significará no solo cambiarse de país, sino que también modificar todas sus redes y sus avances en su trayectoria académica desarrollada en Brasil. Mientras que también existe otro tipo de caso, en que la maternidad elegida en relación a otra persona, que fue la situación de Ignacia, también promueve su responsabilidad con su sobrina, su atención y sus cuidados, pero involucrando también el cambio de hogar desde México a Chile, para poder estar junto a su familia de origen y su sobrina, y con ello volviendo a modificar el rumbo y el desarrollo de su trayectoria académica. Estos tres ejemplos analizados a partir de su visualización en las líneas de tiempo, son un perfecto ejemplo de cómo el uso de esta herramienta puede aportar en las investigaciones con enfoque biográfico.

En general, se puede establecer que el uso de las líneas de tiempo a modo de visualizaciones para comparar casos ha sido de gran utilidad para la investigación. Aquí solo han sido presentados un par de casos que si han visibilizado las diferencias de género entre las trayectorias de académicos y académicas. Por un lado, uno de los beneficios de usar esta herramienta, es que promueve el rescate de forma literal de las emociones, sensaciones o descriptores de las personas entrevistadas, desde la misma voz del sujeto. También permite la visualización desde otra perspectiva de los relatos de vida, siendo que así se le puede mostrar a los/as mismos/as entrevistados/as los avances en distintos encuentros, y así ir avanzando juntos en la co-producción del mismo relato. Además de ello, potencia el carácter interdisciplinario del trabajo en terreno y en el análisis de la información. Así las visualizaciones de las líneas de tiempo se plantean como un útil instrumento visual del relato para el/la investigador/a y la persona entrevistada, pero el/la investigador/a debe considerar con sumo cuidado las expectativas que se tienen sobre esta herramienta, para no caer en generalizaciones, ni en simplificaciones del relato solo en este recurso. Es un complemento de la investigación, y en ningún caso reemplazaría la riqueza del análisis que se logra volviendo al relato (todas las veces que son necesarias), ni la interacción con el/la narrador/a en su contexto. 


\section{Conclusiones}

A través del uso de la visualización de las líneas de tiempo, se ha develado la presencia de diferencias de género entre académicos y académicas. A pesar de que ambos grupos se encontrarán en la misma facultad, estas diferencias son experienciadas por las personas entrevistadas en el día a día. Las cuales se ven acrecentadas en ciertos momentos de la vida, como por ejemplo ya se vio en los resultados, en la tenencia de hijos/as. En el caso de las académicas, ven que su vida familiar y trayectoria académicas se tensionan según el evento. Pero esto mismo no sucedió en el caso de los académicos, quienes de hecho diferenciaban bastante marcadamente ambos aspectos. Incluso, en el caso de los hombres, la trayectoria laboral continúa en su progresivo transcurso en términos de publicaciones, presentaciones en eventos y presencia en investigación. Esto a largo significa una gran diferencia en las trayectorias académicas entre hombres y mujeres.

En este artículo se intentó destacar a la visualización de datos cualitativos, y específicamente las líneas de tiempo, como un elemento investigativo de aporte al enfoque biográfico. Las líneas de tiempo permiten visualizar la temporalidad como herramienta gráfica para el terreno, para el análisis y para la presentación de los datos. La manera de visualizar entrega la posibilidad de que a primera vista la descripción se ordene y simplifique (por medio de los símbolos, o referencias a los años).

Pero uno de sus mayores aportes es la correspondencia mutua e imbricación entre los análisis y la visualización, que no siempre suelen presentarse en los resultados de las investigaciones visualmente, ni en un mismo lugar. La estructura esquemática general de la trayectoria de vida resalta espacios vacíos o de acumulación de eventos, y su ubicación gráfica (comienzo, medio o final de línea). Es decir, el "cuánto" y el "dónde" están los eventos, parece fundamental. De esta forma, la descripción del relato y los análisis, en conjunto con las herramientas de visualización antes mencionadas- relaciones indisolubles-permitieron enriquecer y sostener lo observado en los análisis: Los hombres - investigados en este estudio- significan la paternidad como un evento poco relacionado con lo laboral, cuestión que es fundamental en el análisis de la maternidad de las mujeres. La gran diferencia es que las mujeres viven quiebres y tensiones entre sus trayectorias laborales y familiares, y la biografía del hombre parece estable y progresiva, aun con todos sus matices.

Por otro lado, se demostró que las líneas de tiempo son, en primer lugar, un aporte para rescatar las historias desde la misma voz de los sujetos y descriptores utilizados por ellos. Además, funcionan como un ordenamiento que ayuda a la memoria del entrevistado y el entrevistador y, en última instancia, ayuda a los equipos de investigación. Esto, pues es una forma unificada de trabajar con el material levantado en el terreno coordinando y consensuando ejes relevantes para el análisis. Así el ordenamiento de una narrativa de vida en líneas de tiempo permite a las/os investigadoras/es 
ordenar relatos que los sujetos construyen de manera compleja, asociando diferentes ámbitos de la vida (Coninck \& Godard, 1998). Un aporte relevante, y que debe tenerse en cuenta en la generación de estas líneas, son las transiciones, los puntos de quiebre, la relación con el contexto, entre otros. En el caso de este artículo se utilizaron citas de las entrevistas-en las líneas y el relato-después de este tipo de menciones (por ejemplo, en el cambio que significó tener un hijo para Trinidad).

Es importante considerar las limitaciones y cuidados en el uso de las líneas de tiempo. Creemos necesario destacar que, como todo enfoque cualitativo, el uso de líneas de tiempo no entrega datos generalizados, y en ningún caso reemplaza la riqueza del análisis que se logra volviendo al relato, ni la interacción con el/la narrador/a en su contexto. Esto, pues es el relato quien marcará las temporalidades de las narrativas más allá de la linealidad de la visualización. Por otro lado, y con el sentido de darle continuidad al uso del enfoque biográfico y específicamente a las líneas biográficas de tiempo, quisiéramos relevar la importancia de constituir el registro sistematizado del proceso de producción y divulgación de las líneas de tiempo haciendo énfasis en su relación con los relatos biográficos.

\section{Agradecimientos}

Artículo producido en el marco de los fondos entregados por la ANID PFCHA/DOCTORADO BECAS CHILE 2018 - 72190281 y el proyecto ANID PIA CIE 160007. Las cuatro líneas de tiempo aquí presentadas, se encuentran con mejor resolución en el repositorio abierto de GitHub: https://github.com/FranciscaOrtizRuiz/linea-detiempo-academia.

\section{Referencias}

Arango, Luz Gabriela y Viveros, Mara (1996). Itinerarios profesionales y calendarios familiares: mujeres y hombres en la Gerencia Pública en Colombia. Revista Colombiana de Sociología, III (1): 25-51.

Bernasconi, Oriana (2010). Conocimiento científico y género: la “instalación” de las recién llegadas. Seminario Género y Ciencia. Universidad Alberto Hurtado, Santiago, Chile.

Bernasconi, Oriana (2011). Aproximación narrativa al estudio de fenómenos sociales: principales líneas de desarrollo. Acta Sociológica, 56: 9-36. Doi: http://dx.doi. org/10.22201/fcpys.24484938e.2011.56.28611.

Bertaux, Daniel (1981). Biography and Society. The life history approach in social sciences. Beverly Hills, EEUU: Sage.

Bertaux, Daniel (1989). Los Relatos de vida en el análisis social. Historia y Fuente Oral, 1: 87-96. 
Berríos, Paulina (2005). El sistema de prestigio en las universidades y el rol que ocupan las mujeres en el mundo académico. Calidad en la educación, 23: 349-361. Doi: https://doi.org/10.31619/caledu.n23.301.

Cairo, Alberto (2011). El arte funcional. España: Almut.

Cangiani, Maria Regina y José Roberto Montes (2010). Mulher, maternidade e trabalho academico. Investigación y Educación en Enfermería, 28 (2): 176-186.

Cerros, Elisa y Maria Elena Ramos (2011). El papel de la pareja en el desarrollo profesional. Revista Pequén, 1 (1): 83-92.

Chinchilla, Nuria, Consuelo León, Elizabeth Torres y Miguel Angel Canela (2006). Frenos e impulsores en la trayectoria profesional de las mujeres directivas. Centro Internacional Trabajo y Familia, 632. Recuperado de https://directivasdearagon. es/wp-content/uploads/2011/o7/Frenos-e-impulsores-en-la-trayectoria-profesional-de-las-mujeres-directivas.pdf.

Coninck, Fréderic de y Francis Godard (1998). El enfoque biográfico a prueba de interpretaciones. Formas temporales de causalidad. En Thierry Lulle, Pilar Vargas y Lucero Zamudio (coords.). Los usos de la historia de vida en las ciencias sociales (pp. 250-292). Lima, Perú: Anthropos.

Cole, Jonathan y Helen Zuckerman (1987). Marriage, motherhood and research performance in science. Scientific American, (256): 119-125. Recuperado de http:// www.ncbi.nlm.nih.gov/puvmed/3810117.

Cornejo, Marcela (2006). El Enfoque Biográfico: Trayectorias, Desarrollos Teóricos y Perspectivas. Psyke, 15(1): 95-106. Doi:10.4067/So718-22282006000100oo8.

Cornejo, Marcela, Francisca Mendoza y Rodrigo Rojas (2008). La investigación con relatos de vida: pistas y opciones del diseño metodológico. Psyke, 17(1): 29-39. Doi:10.4067/So718-22282008000100004.

Coulson, Seana y Cristobal Pagán (2014). Understanding Timelines: Conceptual Metaphor and Conceptual Integration. Cognitive Semiotics, 5(1-2): 198-219.

Elder, Glenn H. Jr. (1985). Life course dynamics. Ithaca, New York: Cornell University Press.

England, Paula (2010). The Gender Revolution: Uneven and Stalled. Gender and Society 24(2): 149-166.

England, Paula y George Farkas (1986). Households, employment, and gender: a social, economic and demographic view. New York: New York University.

Entrevista a Agustín, 11 de Agosto del 2014, Chile.

Entrevista a Ignacia, 18 de Agosto del 2014, Chile.

Entrevista a Francisco, 11 de Agosto del 2014, Chile. 
Entrevista a Trinidad, 15 de Octubre del 2014, Chile.

Ferrarotti, Franco (2011). Las historias de vida como método. Acta sociológica, 56: 95119. Recuperado en http://www.redalyc.org/articulo.oa?id=10504402.

Fuentes, Ariel, Jesanm, Cristian, Devoto, Luigi, Angarita, Blanca, Galleguillos, Andrea, Torres Andrea y Mackenna, Antonio (2010). Postergación de la maternidad en Chile: Una realidad oculta. Revista Medicina Chile, 138: 1240-1245. Doi: http:// dx.doi.org/10.4067/So034-98872010001100005.

Gilbert, Jorge (2011). La construcción social del científico. Notas sobre Identidad intelectual y social de comunidades científicas en Chile. Estudios Sociales, 119: 169-2o6. Recuperado de http://www.sistemascomplejos.cl/wp-content/uploads/2012/o4/ La-construcción-social-del-cient\%C3\%ADfico-Jorge-Gibert-Galassi-2011.pdf.

González, Liliana (2010). Análisis de la construcción de trayectorias ocupacionales desde los mecanismos de elección. Revista Colombiana de Sociología, 33(2): 13-29.

Guerra, Dariela y Juan Carlos Skewes (1999). La historia de vida como contradiscurso: pliegues y repliegues de una mujer. Revista Proposiciones, 29: 178-188.

Henderson, Stuart y Eden Segal (2013). Visualizing qualitative data in evaluation research. New Directions for Evaluation, 139: 53-71. Doi:10.1002/ev.20067.

Hiller, Patrick (2011). Visualizing the Intersection of the Personal and the Social Context - The Use of Multi-layered Chronological Charts in Biographical Studies. The Qualitative Report, 16(4): 1018-1033. 1018-1033. Recuperado de https://nsuworks. nova.edu/tqr/vol16/iss $4 / 7$.

Kornblit, Ana Lía (2004). Historias y relatos de vida: una herramienta clase en metodologías cualitativas. En Ana Lía Kornblit, (Eds.) Metodologías cualitativas: modelos y procedimientos de análisis (pp. 15-34). Buenos Aires, Argentina: Biblos.

Knorr Cetina, Karin (2005). La fabricación del conocimiento. Un ensayo sobre el carácter constructivista y contextual de la ciencia. Buenos Aires, Argentina: Universidad Nacional de Quilmes Editorial.

Kyvik, Svein (1990). Motherhood and scientific productivity. Social studies of sciences, (20): 149-16o. Doi: 10.117/o30631290020001005.

Lavados, Jaime (2006). Los negocios universitarios en el mercado del conocimiento. Santiago de Chile: J.C. Sáez Editor.

Longo, Maria Eugenia (2009). Género y tryectorias laborales. Un análisis del entramado permanente de exclusiones en el trabajo. Revista Trayectorias, 11 (28): 118141. Recuperado de http://redalyc.org/articulo.oa?id=60712751008.

Manovich, Lev (2008). La visualización de datos como nueva abstracción y antisublime. Estudios visuales: Ensayo, teoría y crítica de la cultura visual y el arte contemporáneo, 5: 126-135. 
Martínez, Soledad (2012). Ser o no ser: tensión entre familia, subjetividad femenina y trabajo académico en Chile. Un análisis desde la psicología feminista. Revista de Estudios de Género La Ventana, IV (35): 133-163.

Mauro, Amalia (2004). Trayectorias laborales en el sector financiero. Recorridos de las mujeres. Serie Mujer y Desarrollo, 59. CEPAL-Santiago de Chile.

Mason, Mary y Marc Goulden (2004). Marriage and baby blues: redefining gender equity in the academy. Annuals of the American of Political and Social Sciences, 596: 86-103. Doi: 10.1177/0oo2716204268744.

Moyano, Camila y Francisca Ortiz (2016). Los Estudios Biográficos en las ciencias sociales del Chile reciente, hacia la consolidación del enfoque. Revista Psicoperspectivas, 15(1): 17-29. Doi:10.5027/psicoperspectivas-Vol15-Issue1-fulltext-718.

Moyano, Camila y Francisca Ortiz (2018). Subjects analysing subjects in the biographical approach: a generational study of Chilean musicians. Contemporary Social Science, 1-12. Doi: 10.1080/21582041.2018.1448939.

Montilva, Maira (2008). Postergación de la maternidad de mujeres profesionales jóvenes en dos metrópolis latinoamericanas. Revista Utopía y Praxis Latinoamericana, 13 (41): 69-79. Recuperado de http://www.redalyc.org/articulo.oa?id=27904104.

Mora, Claudia (2010). Género, ciencia y mercado laboral. Seminario Género y Ciencia. Universidad Alberto Hurtado, Santiago, Chile.

Munzner, Tamara (2014). Visualization Analysis and Design. Florida: CRC Press.

Ortiz, Francisca (2017). Vida familiar y trayectorias académicas: una aproximación biográfica en una universidad chilena. Revista Polis, 47: 1-19. Doi: 10.4067/So71865682017000200257.

Pujadas, Juan José (2002). El método biográfico: el uso de las historias de vida en ciencias sociales. Madrid, España: Centro de Investigaciones Sociológicas.

Ramos, Claudio (2012). Estructuras de comunicación en el campo de la ciencia social en Chile: un Análisis de redes. Revista REDES, 23 (2): 7-42. Doi: https://doi. org/10.5565/rev/redes.438.

Rosenberg, Daniel y Anthony Grafton (2010). Cartographies of time. New York: Princeton Architectural Press.

Sautu, Ruth (2004). Estilos y prácticas de la investigación biográfica. En R. Sautu (Comp.). El método biográfico. Buenos Aires, Argentina: Ediciones Lumiere.

Selva, Clara, Miguel Angel Sahagún y Susana Pallarès (2011). Estudios sobre Trayectorias Profesional y acceso de la mujer a cargos directivos: un análisis bibliométrico. Revista de Psicología del Trabajo y de las Organizaciones, 27 (3): 227-242. Doi: 10.5093/tr2011v27n3a6. 
Sharim, Dariela (2005). La identidad de género en tiempos de cambio: una aproximación desde los relatos de vida. Psyke, 14(2): 19-32. Doi:10.4067/So71822282005000200002.

Simbürger, Elizabeth y Rosario Undurraga (2013). Jerarquías epistemológicas: Formasde invisibilizar el género en las carreras de sociología en Chile. En C. Mora (Eds.). Desigualdad en Chile: la continua relevancia del género (pp. 171-195). Santiago, Chile: Ediciones Universidad Alberto Hurtado.

Sonnert, Gerhard y Gerald Holton (1996). Career Patterns of Women and Men in the Sciences. American Scientist, 84: 63-71.

Stake, Robert (2013). Estudios de casos cualitativos. En Norman Denzin, y Yvonna Lincoln (Coords.) Las estrategias de investigación cualitativa. (pp. 154-197). España, Gedisa.

Tabin Hasan, Khandaker, Shaugat Abdullah, Sheikh Ahmed, Rezwan y Fausto Giunchiglia (2013). The History of Temporal Data Visualization and a Proposed Event Centric Timeline Visualization Model. International Journal of Computer Applications, 70(27): 27-33. Doi: 10.5120/12241-8497.

Taylor, Steve y Robert Bogdan (200o). Introducción a los métodos cualitativos de investigación. Barcelona, España: Paidós.

Tufte, Edward (2001). The Visual display of quantitative information. Cheshire, CT: Graphics Press. 


\section{Sobre los autores}

Francisca Ortiz Ruiz PhD@ The Mitchell Centre for Social Network Analysis, The University of Manchester. Actualmente se encuentra realizando sus estudios de Doctorado de Sociología, en la Universidad de Manchester. Sus temás de interés son los métodos míxtos (MM y MMSNA), métodos biográficos, análisis de redes sociales, sociología relacional, y gerontología social. Correo Electrónico: franortizruiz@gmail. com o francisca.ortiz@manchester.ac.uk. iD https://orcid.org/0000-0001-8538-4688

Camila Moyano Dávila PhD. Actualmente, trabaja en el Centro Justicia Educacional de la Pontificia Universidad Católica de Chile, y sus temas de interés son, los métodos cualitativos avanzados, teorías críticas sobre educación, minorías en contextos educativos y justicia educacional. Autora correspondiente. Correo Electrónico: camila.moyano@uc.cl. (iD) https://orcid.org/0000-0002-6357-3469

Daniela Moyano Dávila es diseñadora, se encuentra cursando el último año del Magíster en Sociología de la Pontificia Universidad Católica de Chile. Su tema de interés principal es la diseño de información y visualización de Datos, área en la que se ha desempeñado como docente. Correo Electrónico: dpmoyano@uc.cl. (D http:// orcid.org/0000-0002-3454-1070 


\title{
CUHSO
}

Fundada en 1984, la revista CUHSO es una de las publicaciones periódicas más antiguas en ciencias sociales y humanidades del sur de Chile. Con una periodicidad semestral, recibe todo el año trabajos inéditos de las distintas disciplinas de las ciencias sociales y las humanidades especializadas en el estudio y comprensión de la diversidad sociocultural, especialmente de las sociedades latinoamericanas y sus tensiones producto de la herencia colonial, la modernidad y la globalización. En este sentido, la revista valora tanto el rigor como la pluralidad teórica, epistemológica y metodológica de los trabajos.

\author{
EDITOR \\ Matthias Gloël \\ CoORdinadora EDITORIAL \\ Claudia Campos Letelier \\ Corrector de ESTILO Y DiSEÑAdor \\ Ediciones Silsag \\ Traductor, CORRECTOR LENGUA INGLESA \\ Aurora Sambolin Santiago \\ SITIO WEB \\ cuhso.uct.cl \\ E-MAIL \\ cuhso@uct.cl
}

LICENCIA DE ESTE ARTÍCULO

Creative Commons Atribución Compartir Igual 4.0 Internacional 